\title{
Expression of $\mathrm{CD}_{133}{ }^{+}$cancer stem cells in childhood malignant melanoma and its correlation with metastasis
}

\author{
Rola Al Dhaybi ${ }^{1,2}$, Hervé Sartelet ${ }^{2}$, Julie Powell ${ }^{1}$ and Victor Kokta ${ }^{2}$ \\ ${ }^{1}$ Division of Dermatology, CHU Sainte-Justine, University of Montreal, Montreal, QC, Canada and \\ ${ }^{2}$ Department of Pathology, CHU Sainte-Justine, University of Montreal, Montreal, QC, Canada
}

\begin{abstract}
Cancer stem cells expressing CD133 exist in a wide array of tumors and their identification in malignant melanoma may help refine classification, diagnosis and treatment. To study the correlation between CD133 expression in childhood melanoma and lymph node and/or visceral metastasis, we evaluated 12 cases of malignant melanoma and 12 control cases of Spitz nevus occurring in children. Double immunostaining with CD133 and Ki-67 was performed in the cases showing CD133 positivity. Three melanoma patients had lymph node metastasis and only one had multivisceral metastases; CD133 was positive only in these four patients. The $\mathrm{Ki}-67$ index was lower in the $\mathrm{CD}^{-133^{+}}$cells in comparison with the CD133- melanoma cells in three cases. We found no positivity for CD133 in all the Spitz nevi. CD133 ${ }^{+}$cancer stem cell expression in childhood malignant melanoma might correlate with lymph node and/or visceral metastasis and may have a low proliferative $\mathrm{Ki}-67$ index that might explain their chemoresistance.

Modern Pathology (2010) 23, 376-380; doi:10.1038/modpathol.2009.163; published online 8 January 2010
\end{abstract}

Keywords: CD133; Ki-67; malignant melanoma; Spitz; childhood

Cancer stem cells, derived from clonal expansion of atypical cells, exist in a wide array of tumors and are becoming increasingly important to understand the molecular mechanisms that regulate self-renewal, differentiation and progression of metastasis. ${ }^{1-3}$ Identification of cancer stem cells may help refine classification, diagnosis and treatment of cancers, including malignant melanoma. ${ }^{4}$ New therapies targeting these cells, which are fundamental for tumor progression, could significantly improve the response to treatment in malignant melanoma. Therefore, it is of paramount importance to identify subpopulations of cells in malignant melanoma, exhibiting significant differences in terms of proliferation, stem cell marker expression and behavior. ${ }^{1}$ CD133 (Prominin-1) is considered the most important cancer stem-cell-associated marker iden-

Correspondence: Dr R Al Dhaybi, MD, Department of Pathology, CHU Sainte-Justine, University of Montreal, 3175 Chemin CôteSainte-Catherine, Montreal, QC H3T 1C5, Canada.

E-mail: roladhaybi@yahoo.com

Received 16 June 2009; revised 6 October 2009; accepted 7 October 2009; published online 8 January 2010 tified so far, with increased expression in the cancer stem cell fraction of a large variety of human malignancies, including malignant melanoma. ${ }^{2}$

Cutaneous malignant melanoma is a rare disease in childhood and adolescence, constituting only $2 \%$ of melanomas and accounting for $3 \%$ of malignancies in patients younger than 20 years. ${ }^{5}$ Spitz nevus, previously named benign juvenile melanoma by Sophie Spitz, is a benign acquired proliferation of melanocytes, commonly presenting in childhood with histopathological features that sometimes overlap with those of malignant melanoma. ${ }^{6,7}$ Klein et $a l^{4}$ observed significant increases in the expression of the stem cell markers CD133, CD166 and nestin in primary and metastatic melanoma in comparison with their expression in banal nevi.

CD133, directly or through its interaction with signaling pathways, influences cell polarity, migration and interaction of cancer stem cells with the surrounding cells and the extracellular matrix, contributing to the metastatic potential. ${ }^{2}$ This study is, to the best of our knowledge, the first to evaluate CD133 expression in childhood malignant melanoma and Spitz nevus. 


\section{Materials and methods}

Twelve pathological specimens from formalin-fixed, paraffin-embedded skin biopsies of patients with diagnosis of malignant melanoma were obtained from the archives of the Department of Pathology of our tertiary children hospital, CHU Sainte-Justine. All cases were either consultations or referrals. The same histopathological criteria used for diagnosing adult melanoma were applied for our cases of childhood melanoma. The majority of childhood melanomas were of nodular subtype, often presenting spitzoid histological features. The most useful histopathological criteria for differentiating nodular childhood melanoma from Spitz nevus are (1) lack of dermal maturation, (2) deep dermal mitoses, (3) numerous dermal mitoses, (4) atypical dermal mitoses and (5) abundant and lateral pagetoid ascension. ${ }^{5}$ Moreover, all childhood melanoma cases were reviewed and confirmed by at least two dermatopathologists. A control group of 12 formalin-fixed, paraffin-embedded skin biopsies of patients having the diagnosis of Spitz nevus were also collected. All patients were younger than 18 years and had their biopsies done between 1990 and 2008. Spitz nevus patients were chosen as a control group because malignant melanoma can have similar features to both classic and atypical Spitz nevi and must be ruled out in all of these cases. ${ }^{7}$

Collection of data from malignant melanoma and Spitz nevus patients' charts was carried out. The age at diagnosis, sex, location, histological diagnosis with Clark staging and Breslow depth for malignant melanoma, presence of metastasis or recurrence, duration of follow-up and evolution after diagnosis were noted. One of the malignant melanoma patients had no follow-up in our hospital and another one did not have enough pathology specimen for further evaluation and both of them were excluded from our study.

\section{Immunohistochemical Staining}

Immunohistochemical double staining method was performed on paraffin-embedded sections using
ultraView Universal Alkaline Phosphatase Red Detection Kit (Ventana; Ventana Medical System, Tucson, AZ, USA) after 30 min of antigen retrieval in $95^{\circ} \mathrm{C}$ heated buffer solution, $60 \mathrm{~min}$ of exposition to CD133 (Abcam, Cambridge, MA, USA) and $16 \mathrm{~min}$ of signal amplification. Then the ultraView Universal DAB Detection Kit (Ventana; Ventana Medical System) was used after $30 \mathrm{~min}$ of heat-based antigen retrieval, $16 \mathrm{~min}$ of exposition to an antibody against Ki-67 (Neomarkers, Lab Vision, Fremont, CA, USA) and $16 \mathrm{~min}$ of signal amplification. Single immunohistochemical staining method omitted the second step, using either the ultraView DAB Detection Kit or the ultraView Universal Alkaline Phosphatase Red Detection Kit.

CD133 expression was evaluated in all specimens of primary malignant melanoma and/or their metastases or recurrence, and in all specimens of Spitz nevus. Double immunostaining with CD133 and Ki67 was performed in the cases showing CD133 positivity (Tables 1 and 2).

\section{Results}

Table 1 shows the malignant melanoma cases with their characteristics and their CD133 expression. Seven malignant melanoma patients were girls and 3 were boys. The mean age of these patients was 10 years. Six of the malignant melanoma were located on the lower limb. Lower extremities are known to be the most common primary site of malignant melanoma in childhood. ${ }^{8}$ Most of the patients ( 7 of 10) had a Clark IV stage of their malignant melanoma. Two of the malignant melanoma patients died within 1 year of diagnosis due to progression of their disease. Three patients had lymph node metastasis and only one had multivisceral metastases. CD133 was only positive in these four patients in our study. CD133 positivity was either diffuse or focal in the primary malignant melanoma cases (Figure 1) and was focally positive in the metastases cases (Figure 2). In case 10, the CD133 was more positive in the lymph node metastasis in comparison with the primary malignant melanoma.

Table 1 Characteristics of patients with childhood malignant melanoma

\begin{tabular}{|c|c|c|c|c|c|c|}
\hline Case/Sex & $\begin{array}{c}\text { Age at diagnosis } \\
\text { (years) }\end{array}$ & Location & $\begin{array}{l}\text { Clark staging/ } \\
\text { Breslow (mm) }\end{array}$ & Metastasis & Follow-up time & $C D 133$ \\
\hline $1 / F$ & 14 & Scalp & $\mathrm{IV} / 2.38$ & No & 6 months & - \\
\hline $2 / \mathrm{F}$ & 16 & Ankle & IV/1.33 & No & 15 months & - \\
\hline $3 / \mathrm{M}$ & 9 & Thigh & $\mathrm{I} / 1$ & No & 7 months & - \\
\hline $4 / \mathrm{M}$ & 5 & Leg & $\mathrm{IV} / 4.7$ & Lymph node & 60 months & + \\
\hline $5 / \mathrm{F}$ & 12 & Back & $\mathrm{IV} / 5.58$ & Multivisceral & Dead 25 months after diagnosis & + \\
\hline $6 / F$ & 4 & Foot & IV/6 & No & 84 months & - \\
\hline $7 / \mathrm{M}$ & 12 & Trunk & IV/2.5 & No & 36 months & - \\
\hline $8 / \mathrm{F}$ & 12 & Foot & $\mathrm{III} / 0.8$ & No & 84 months & - \\
\hline $9 / F$ & 8 & Knee & IV $/ 4.4$ & Lymph node & 4 months & + \\
\hline $10 / \mathrm{F}$ & 10 & Shoulder & III/1.9 & Lymph node & Dead 17 months after diagnosis & + \\
\hline
\end{tabular}

F, female; M, male. 


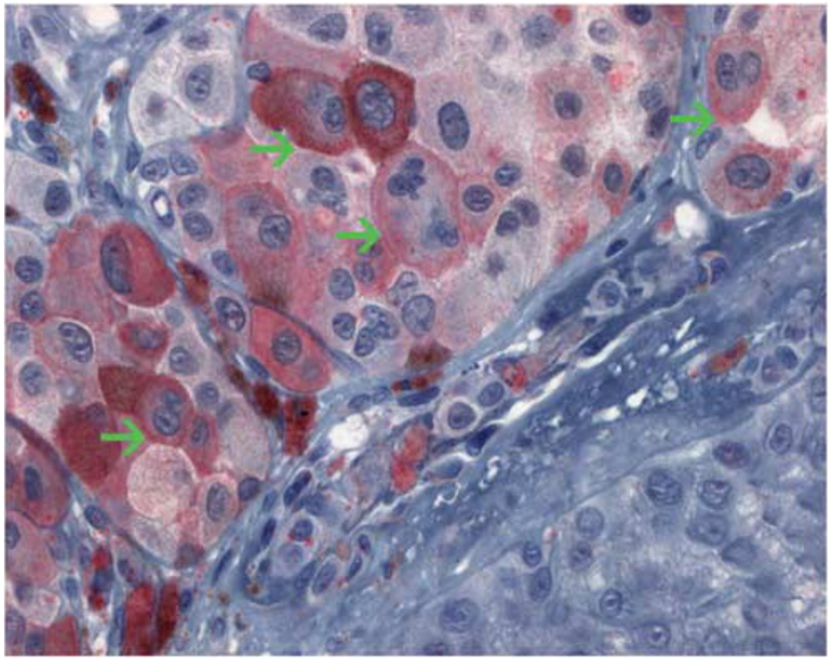

Figure 1 Positive CD133 (red) in multifocal melanoma cells, revealing clear cytoplasmic staining with focal membranous re-enforcement (green arrow). Original magnification, $\times 400$.

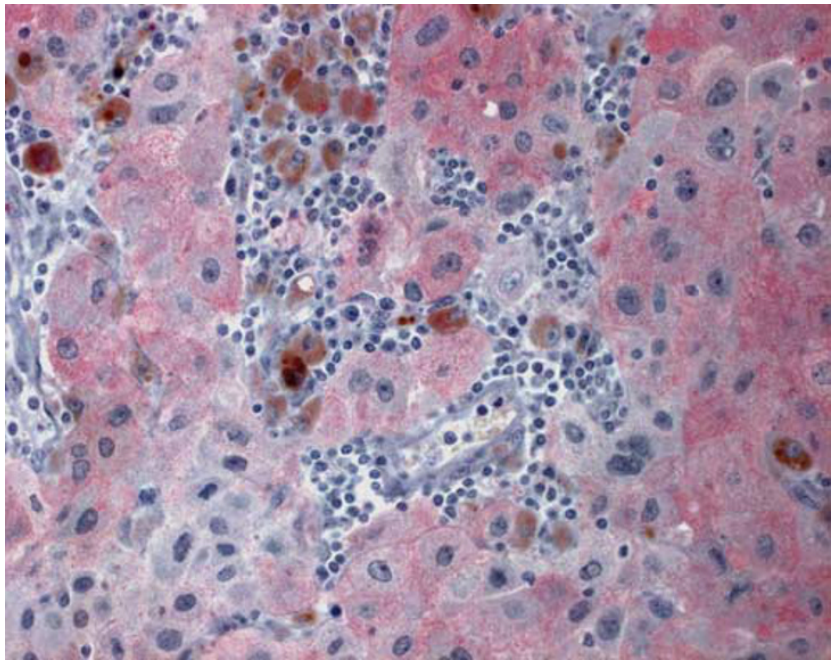

Figure 2 Melanoma cells with mostly cytoplasmic positive CD133 staining (red) within a lymph node. Original magnification, $\times 400$.

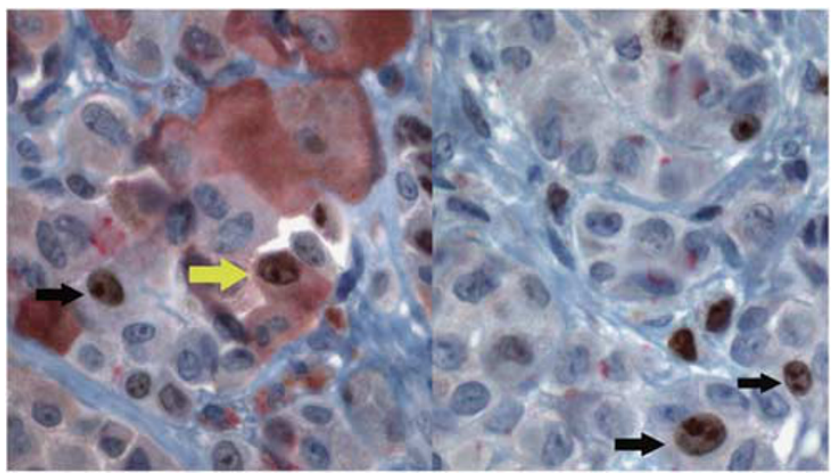

Figure 3 CD133 (red)-Ki-67(dab) double staining revealing a lower proportion of $\mathrm{CD} 133^{+} / \mathrm{Ki}-67^{+}$cells (yellow arrow) compared with $\mathrm{CD} 133^{-} / \mathrm{Ki}-67^{+}$melanoma cells (black arrow). Original magnification, $\times 400$.
Table 2 Frequency of Ki-67+ nuclei in $\mathrm{CD}^{+} 133^{-}$and $\mathrm{CD} 133^{+}$cells of the malignant melanoma cases having positive staining for CD133

\begin{tabular}{lcc}
\hline $\begin{array}{l}\text { Patient case number } \\
\text { based on Table 1 }\end{array}$ & $\begin{array}{c}\text { Ki-67 } \\
\text { CD133- }\end{array}$ & $\begin{array}{c}K^{+}-67^{+} \\
C D 133^{+}(\%)\end{array}$ \\
\hline 4 & 32 & 3 \\
5 & 18 & 4 \\
9 & 13 & 2 \\
10 & 66 & 60 \\
\hline
\end{tabular}

Table 3 Characteristics of patients with Spitz nevi

\begin{tabular}{lclc}
\hline $\begin{array}{c}\text { Case/sex } \\
\text { Age at diagnosis } \\
\text { (years) }\end{array}$ & Location & CD133 \\
\hline 1/M & 3 & Cheek & - \\
$2 / \mathrm{M}$ & 4 & Cheek & - \\
$3 / \mathrm{M}$ & 10 & Ear & - \\
4/F & 6 & Finger & - \\
5/F & 9 & Knee & - \\
$6 / \mathrm{M}$ & 14 & Thigh & - \\
$7 / \mathrm{M}$ & 6 & Face & - \\
8/M & 0.5 & Scalp & - \\
9/M & 10 & Leg & - \\
10/M & 3 & Cheek & - \\
11/F & 6 & Abdomen & - \\
12/M & 1 & Leg & - \\
\hline
\end{tabular}

F, female; M, male.

CD133/Ki-67 double immunostaining (Figure 3) of three of the four $\mathrm{CD}_{133^{+}}$cases (cases 4, 5 and 9) showed a Ki-67 index that was lower in the CD133 ${ }^{+}$ melanoma cells in comparison with the Ki-67 index in the CD133- cells (Table 2). In the fourth case (case number 10), the difference between the Ki-67 index in the $\mathrm{CD}_{133^{+}}$and the $\mathrm{CD} 133^{-}$cells was less obvious.

Table 3 shows the Spitz nevus patients with their characteristics. Nine patients were girls and three were boys. The mean age of these patients was 6 years. Half of the lesions (6 of 12) were located on the head whereas 4 were located on the lower extremity. This finding is concordant with the medical literature, in fact most Spitz nevi are known to occur on the face and lower extremities. ${ }^{6}$ There was no positivity for CD133 in all of the Spitz nevus cases. Moreover, all of the Spitz nevi behaved in a benign manner and did not recur.

\section{Discussion}

Cancers originate from cancer stem cells or tumor stem cells. ${ }^{3}$ Cancer stem cells have been defined in a variety of human and mouse tumors. ${ }^{9}$ These cells represent only a small fraction of cancer that possesses the capability to regenerate cancer whereas most other cancer cells lack this 
regenerative capability. ${ }^{3}$ Expression of stem cell markers and the isolation of stem-like cells has recently been described in malignant melanoma. Whether melanoma stem cells are derived from melanocyte stem cells, melanocyte progenitors or more mature melanocytes that have dedifferentiated remains unclear. ${ }^{7}$ Subpopulations expressing CD20, CD166, nestin and CD133 have been identified in melanoma tissues. ${ }^{4,9}$ A significantly greater percentage of malignant melanoma contained stem cells that expressed CD166, CD133 and nestin in comparison with the stem cells of banal nevi. However only nestin showed a statistical difference when comparing primary and metastatic melanomas. ${ }^{4}$

CD133 is a transmembrane glycoprotein also known as prominin-1 because of its prominent location on the protrusion of cell membranes. ${ }^{2,9}$ CD133 is normally expressed on undifferentiated cells including endothelial progenitor cells, hematopoietic stem cells, fetal brainstem cells and prostate epithelial cells. ${ }^{9}$ Its interest as a cancer stem cell marker has grown dramatically since the theory suggesting that it was able to identify a cancer-initiating subpopulation in the brain and colon. CD $133^{+}$cells have also been found in acute and chronic myeloid leukemias, lymphoblastic lymphoma, hepatocarcinoma, prostate, pancreatic cancer, osteosarcoma and melanoma. ${ }^{1,4,9}$

The role of CD133 protein as a factor serving in directing tumor growth is the subject of active scrutiny. Shmelkov et $a l^{10}$ found that $\mathrm{CD}^{133^{-}}$cells are capable of tumorigenesis in colon cancer in mice and produce even more aggressively growing tumors than the tumors with $\mathrm{CD}_{133^{+}}$cells. According to the experiment by Zabierowski et al, less than 1\% of cells within metastatic melanoma express CD133. However, only the CD133 ${ }^{+}$cells were able to induce tumors in nonobese diabetic/severe combined immunodeficiency mice, whereas the CD133fraction failed to regenerate tumors, indicating that only a small percentage of cells were capable of recapitulating tumor growth. ${ }^{9}$ Cells with stable downregulation of CD133 showed decreased metastatic capacity in vivo, suggesting that CD133 is involved in the tumor cell's capacity to seed to distant sites. The residual expression of CD133 may account for the remaining metastatic capacity. ${ }^{2}$ Another study showed that cells expressing CD133 or CD44 or both had stronger tumorigenic potential in mice than those without these molecules expression. These cells generated tumors faster and resulted in higher mortality in mice. ${ }^{3}$ Melanoma cancer stem-cell surface molecules could be identified in melanoma biopsies and could be used as prognostic indicators. CD133 expression might be associated with an increased risk of metastasis and a worse outcome in childhood malignant melanoma. In fact, our study showed that all malignant melanoma patients, who had associated metastasis, had a positive expression of CD133. Moreover, we found stronger CD133 positivity in the metastatic lesion in comparison with the primary melanoma in case 10. The lack of CD133 positivity in all our Spitz nevus cases could be related to the benign behavior of these nevi. It is sometimes difficult to distinguish Spitz nevus from malignant melanoma and many investigators are searching for tools and techniques that may help enhance diagnostic accuracy. ${ }^{7}$ CD133 expression might be a useful tool to suggest a malignant behavior.

The most widely used proliferation marker in pathology is Ki-67, a nuclear antigen present in all active phases of cell-cycle proliferation $\left(G_{1}, S, G_{2}\right.$ and $M$ ) but absent in the quiescent phase $\left(G_{0}\right)$. Ki-67 staining is reported as positive in $13-30 \%$ of the cells in a malignant melanoma. There have been mixed results using $\mathrm{Ki}-67$ as a prognostic marker in malignant melanoma. Some studies have shown an independent positive correlation between Ki-67 staining and metastatic potential and mortality only in thick melanomas; others have shown a similar correlation only in thin melanomas. Many studies have shown an increase in recurrence rate and mortality that directly correlates with increasing positivity for Ki-67; however, others have shown that this correlation is not independent of Breslow thickness or did not find any correlation at all between them. ${ }^{11}$ Presence of CD133 ${ }^{+} / \mathrm{Ki}-67^{+}$cells was considered a prognostic factor of disease progression and poor clinical outcome in glioblastoma multiforme patients. ${ }^{12}$ In three of the four $\mathrm{CD}_{133^{+}}$malignant melanoma cases, the comparatively lower Ki-67 index in the cancer stem cells may reflect a slower proliferation of these specific cells, possibly explaining their chemoresistance. The fourth $\mathrm{CD} 133^{+}$malignant melanoma case (case 10) had Ki-67 indexes that were 2-3 times higher in both $\mathrm{CD}_{133^{+}}$and $\mathrm{CD}_{133^{-}}$melanoma cells in comparison with the ones of the melanoma cells of the other three $\mathrm{CD}_{133}{ }^{+}$cases (cases 4, 5 and 9). Case 10 was the most aggressive melanoma in our series and led to the death of the patient 17 months after diagnosis. The high Ki-67 index might reflect a fast tumor progression in this case (Tables 1 and 2).

The clinical relevance of targeting cancer stemcell-associated genes per se is supported by recent studies targeting CD24 for treatment of colon and pancreatic cancer, and targeting CD133 by antibodydrug conjugates for the treatment of hepatocellular and gastric cancers. ${ }^{2}$ Owing to the potential importance of $\mathrm{CD}_{133^{+}}$cell populations as donor cells for cell therapy and of targeting CD133 as an anticancer strategy, a close monitoring of the proliferative activity of these cells will be required in future clinical applications. ${ }^{2}$ ABCB5, a novel drug transporter and chemoresistance mediator, was coexpressed with CD133 in distinct tumor subpopulations in both cultured melanoma cells and clinical melanoma tumors. Tumor growth and metastasis driven by chemoresistant cancer stem cells might explain the failure of existing therapies to eradicate solid tumors consistently. ${ }^{4}$ Recently, 
hepatocellular carcinoma $\mathrm{CD}_{133^{+}}$cancer stem cells were found to confer chemoresistance by preferential expression of survival proteins involved in the Akt/PKB and Bcl-2 pathway; the activation of the Akt/PKB pathway being a regulator of cellular processes including apoptosis, proliferation, differentiation and metabolism. Therefore, the Akt pathway can be a target for novel antineoplastic therapies. ${ }^{13}$ Furthermore, CD133 stem cell may pose as target for antibody-based therapies that would selectively eliminate these cells. ${ }^{9}$

In conclusion, our study showed that $\mathrm{CD} 133^{+}$ cancer stem cell expression in childhood malignant melanoma might correlate with lymph node and/or visceral metastasis. These CD133 ${ }^{+}$cancer stem cells may have a low proliferative Ki-67 index that might explain their chemoresistance. Further studies involving melanoma cancer stem cell lines could be useful for evaluating the importance of the P13K/ Akt signaling pathway activation in these cells. Targeting this pathway by specific inhibitors could decrease the chemoresistance of cancer stem cells in childhood malignant melanoma.

\section{Disclosure/Conflict of interest}

The authors declare no conflict of interest.

\section{References}

1 Tirino V, Desiderio V, D’Aquino R, et al. Detection and characterization of $\mathrm{CD} 133^{+}$cancer stem cells in human solid tumours. PLoS ONE 2008;3:e3469.

2 Rappa G, Fodstad O, Lorico A. The stem cellassociated antigen CD133 (Prominin-1) is a molecular therapeutic target for metastatic melanoma. Stem Cells 2008;26:3008-3017.

3 Zabierowski SE, Herlyn M. Melanoma stem cells: the dark seed of melanoma. J Clin Oncol 2008;26: 2890-2894.

4 Klein WM, Wu BP, Zhao S, et al. Increased expression of stem cell markers in malignant melanoma. Mod Pathol 2007;20:102-107.

5 Jafarian F, Powell J, Kokta V, et al. Malignant melanoma in childhood and adolescence: a report of 13 cases. J Am Acad Dermatol 2005;53:816-822.

6 Shaffer JV. Pigmented lesions in children: when to worry. Curr Opin Pediatr 2007;19:430-440.

7 Sulit DJ, Guardiano RA, Krivda S. Classic and atypical Spitz nevi. Review of the literature. Cutis 2007;79: 141-146.

8 Ferrari A, Bono A, Baldi M, et al. Does melanoma behave differently in younger children than in adults? A retrospective study of 33 cases of childhood melanoma from a single institution. Pediatrics 2005;115:649-654.

9 Dou J, Pan M, Wen P, et al. Isolation and identification of cancer stem-like cells from murine melanoma cell lines. Cell Mol Immunol 2007;4:467-472.

10 Shmelkov SV, Butler JM, Hopper AT, et al. CD133 expression is not restricted to stem cells, and both $\mathrm{CD}_{133^{+}}$and $\mathrm{CD}_{133^{-}}$metastatic colon cancer cells initiate tumor. J Clin Invest 2008;118:2111-2120.

11 Ohsie SJ, Sarantopoulos GP, Cochran AJ, et al. Immunohistochemical characteristics of melanoma. J Cut Pathol 2008;35:433-444.

12 Pallini R, Ricci-Vitiani L, Banna GL, et al. Cancer stem cell analysis and clinical outcome in patients with glioblastoma multiforme. Clin Cancer Res 2008;14: 8205-8212.

13 Ma S, Lee TK, Zheng BJ, et al. CD133 ${ }^{+}$HCC cancer stem cells confer chemoresistance by preferential expression of the Akt/PKB survival pathway. Oncogene 2008;27:1749-1758. 